Meta

Journal des traducteurs

Translators' Journal

\title{
BAUNEBJERG HANSEN, Gitte (1990) : Artikelstruktur im zweisprachigen Wörterbuch, Tübingen, Niemeyer, Coll. Lexicographica Series Maior 35, VIII + 196 p.
}

\section{André Clas}

Volume 37, numéro 3, septembre 1992

URI : https://id.erudit.org/iderudit/002318ar

DOI : https://doi.org/10.7202/002318ar

Aller au sommaire du numéro

\section{Éditeur(s)}

Les Presses de l'Université de Montréal

\section{ISSN}

0026-0452 (imprimé)

1492-1421 (numérique)

Découvrir la revue

Citer ce compte rendu

Clas, A. (1992). Compte rendu de [BAUNEBJERG HANSEN, Gitte (1990) : Artikelstruktur im zweisprachigen Wörterbuch, Tübingen, Niemeyer, Coll. Lexicographica Series Maior 35, VIII + 196 p.] Meta, 37(3), 561-562.

https://doi.org/10.7202/002318ar d'utilisation que vous pouvez consulter en ligne.

https://apropos.erudit.org/fr/usagers/politique-dutilisation/ 
BAUNEBJERG HANSEN, Gitte (1990): Artikelstruktur im zweisprachigen Wörterbuch, Tübingen, Niemeyer, Coll. Lexicographica Series Maior 35, VIII + 196 p.

L'auteur analyse une dizaine de dictionnaires bilingues, danois-allemand, allemanddanois, et constate, dans la majorité des cas, un «chaos» (c'est son mot) dans l'organisation des divers articles. En s'appuyant sur diverses publications de lexicographie bilingue et sur ses analyses et réflexions personnelles, l'auteur propose un schéma pour les articles de dictionnaires bilingues. Son plaidoyer est illustré d'entrées dictionnairiques modèles représentant cinq parties du discours (pp. 142 à 186) en allemand et en danois.

Le but défini par l'auteur part de la citation en allemand de F.-J. Hausmann qui déclare qu'«un dictionnaire est une collection d'unités lexicales présentées par un médium déterminé donnant, pour un usager défini, certaines informations de telle façon qu'un accès rapide à un renseignement est possible» (notre traduction). L'analyse de divers dictionnaires montre que dans la majorité des cas, ces principes ne sont pas respectés. L'usager perd un temps infini à retrouver des informations, car leur présentation est déficiente, inégale, désordonnée et variable d'un article à l'autre et d'un dictionnaire à l'autre. L'auteur reprend ensuite le plaidoyer général pour la typologie dictionnairique apparue il y a une vingtaine d'années et qui veut que l'on développe des dictionnaires bilingues dans un cadre où les principes «actif-passif» soient rigoureusement appliqués. Il s'agit en fait de l'opposition que l'on fait souvent entre «décodage» et «encodage» ou encore de «dictionnaires de version» et de «dictionnaires de thème», de «dictionnaire actif» et de «dictionnaire passif». Il est certain que le problème se pose de façon théorique. Mais il y a de nombreux obstacles, la commercialisation n'étant pas le moindre, puisqu'il faut produire des dictionnaires différents pour chaque groupe d'usagers très précis dont les besoins sont homogènes. On doit ainsi envisager des dictionnaires pour des élèves de telle ou telle année scolaire. Cela est tout à fait réalisable dans cette limite, mais cela nous semble déjà un peu plus problématique lorsqu'il s'agit d'un public plus général, donc plus disparate.

L'auteur analyse les divers problèmes que soulèvent les moyens de détermination de différenciation de la signification et note avec justesse qu'il ne faut pas l'assimiler à l'explication de la signification dans un dictionnaire unilingue. Une orientation de la signification ne doit apparaître dans le dictionnaire bilingue que si elle joue un rôle de différenciateur ou de compensateur. Le choix des exemples n'est pas non plus sans embûches. L'exemple doit être considéré comme faisant partie intégrante d'une catégorie lexicale et doit apporter une information supplémentaire à l'équivalent. Pour les collocations figées, ce que l'on appelle souvent des phrasèmes, une glose est beaucoup plus appropriée qu'un exemple, car il ne faudrait pas donner l'illusion d'une utilisation non restrictive et analogique. Toute la question des collocations soulève d'ailleurs des problèmes liés à la syntaxe. Il faut soigneusement distinguer entre des formations libres et ce 
que l'on appelle des fonctions lexicales. On peut d'ailleurs souvent se demander quelles limites à la liberté nous imposent les combinaisons dans le choix des mots. Si, à titre d'exemple, j'analyse le mot «évidence», les seules possibilités de choix de verbes sont: «aller contre $l^{\prime} \ldots$, apparaitre à $l^{\prime} \ldots$, démontrer à $l^{\prime} \ldots$, être $l^{\prime} \ldots$ même, être en ..., mettre (qqc.) en ..., se mettre en ..., nier l'..., refuser à l'.., se rendre à l'...».

L'auteur démontre à l'aide d'exemples bien choisis les neuf différentes thèses à considérer pour rédiger un dictionnaire bilingue. (1) Le groupe cible des utilisateurs doit être déterminé de façon aussi précise que possible, leurs besoins et leurs attentes sont une partie indispensable de la préparation d'un projet de dictionnaire. (2) Les besoins et les attentes des utilisateurs orientent le choix des informations à présenter dans le dictionnaire. (3) Les objectifs du dictionnaire (encodage ou décodage, version ou thème) déterminent les informations spécifiques fournies. (4) La suppression radicale de l'information sur la langue maternelle, sous prétexte que chaque locuteur a une bonne compétence dans sa langue maternelle, ne correspond en aucun cas à la réalité constatée. (5) L'ordre et la présentation des équivalents doivent être conditionnés par les relations entre le lemme et les équivalents et non pas, comme dans des dictionnaires unilingues, par les structures sémantiques de l'entrée. (6) Les différences sémantiques doivent être présentées dans la langue maternelle de l'utilisateur. (7) Des règles de rédaction pour la présentation de l'information donnée dans la microstructure doivent assurer l'uniformité des articles du dictionnaire. (8) Les principes de présentation des articles doivent être explicités et exemplifiés dans la préface. (9) Les informations fournies dans le dictionnaire doivent être explicites.

Nous voudrions recommander la lecture de cet ouvrage clair, bien écrit, bien documenté et bien exemplifié à la fois aux ufaiseurs» de dictionnaires et aux usagers de dictionnaires en général. Les uns pourront mieux faire leur travail et les autres pourront mieux comprendre le travail fait et donc se servir plus efficacement d'un type d'ouvrage indispensable.

ANDRÉ CLAS 